\title{
Gene expression profiling of the mouse gut: Effect of intestinal flora on intestinal health
}

\author{
WENHUA ZHU ${ }^{1,2^{*}}, \mathrm{JUN} \mathrm{LI}^{1 *}$ and BENYAN WU ${ }^{1}$ \\ ${ }^{1}$ Department of Gastroenterology, South Building, Chinese PLA General Hospital, Beijing 100853; \\ ${ }^{2}$ Department of Oncology, The 309th Hospital of Chinese PLA, Beijing 100091, P.R. China
}

Received August 9, 2016; Accepted May 12, 2017

DOI 10.3892/mmr.2017.8298

\begin{abstract}
The present study aimed to investigate the molecular mechanisms, including potential genes, pathways and interactions, underlying the effect of intestinal flora on intestinal health. The gene expression profiles of GSE22648 were downloaded from the Gene Expression Omnibus database to screen differentially expressed genes (DEGs). The Database for Annotation, Visualization and Integrated Discovery was used for Gene Ontology (GO) functional and pathway enrichment analysis of the DEGs. DEG-associated literature was mined using the GenCLip 2.0 online tool. Finally, GO and pathway enrichment analyses of the DEGs in the literature were processed. By comparing microbiota-depleted mouse samples and control mouse samples, a total of 115 DEGs, including 58 upregulated genes and 57 downregulated genes, were screened. The upregulated genes were enriched into various GO terms, including microsome, oxidation reduction and heme binding, whereas the 57 downregulated DEGs were enriched in different functions, including DNA packaging and linoleic acid metabolism. A total of 19 genes, including baculoviral IAP repeat containing 5, aurora kinase $\mathrm{A}$, angiotensin I converting enzyme 2 and free fatty acid receptor 2 were identified and enriched in four modules, including cell division, chromosome segregation, inflammatory bowel disease and inflammatory response. AURKA, inner centromere protein antigens $135 / 155 \mathrm{kDa}$, baculoviral IAP repeat containing 5, aurora kinase B and solute carrier family 22 (organic cation/zwitterion transporter) member 4 were identified as potential important genes for intestinal flora and intestinal disease treatment through their involvement in various functions, including cell division, chromosome segregation, inflammatory bowel disease and inflammatory response.
\end{abstract}

Correspondence to: Dr Benyan Wu, Department of Gastroenterology, South Building, Chinese PLA General Hospital, 28 Fuxing Road, Beijing 100853, P.R. China

E-mail: wushshhdfhff@hotmail.com

${ }^{*}$ Contributed equally

Key words: intestinal flora, intestinal health, target genes

\section{Introduction}

With improvements in living standards, increasing attention is focused on intestinal health. Intestinal health is defined as a state, in which there are no signs of gut disease, no risk of intestinal disease and no intestinal diseases (1). The adhesion of intestinal flora to the intestinal wall can have a beneficial or deleterious role in hosts, and determines the health status of the gut (2). Therefore, investigations of intestinal flora are essential for intestinal health. A previous study showed that the diversity of the colonic mucosa was closely associated with bacterial microflora in patients with active inflammatory bowel disease (3). Disturbances of microbiota may also induce immune dysregulation and can develop into inflammatory bowel disease (4). In addition, gut bacteria can degrade various xenobiotics, including halogenated aromatic compounds, which may be beneficial for human health (5).

Various genes associated with intestinal flora have been identified for investigating intestinal health. In the microbial community, the diversity of genes can provide several types of enzymes and pathways associated with intestinal health (6). Jia et al (7) suggested that cytochrome P450, family 4, subfamily F, polypeptide 14 and tachykinin, precursor 1 interact with each other and are involved in pathways, including the neuropeptide signaling pathway, oxidation reduction and metabolism by regulating intestinal microbiota depletion. In addition, FBJ murine osteosarcoma viral oncogene homolog has been confirmed to enhance gut health by altering the ecology of the gut microbiota and improving the proteolysis of feces (8). Another important gene, interleukin 6 has been confirmed as a key molecule in gut barrier dysfunction (9). Although several genes associated with intestinal health have been investigated, further information is required. Therefore, molecular mechanisms, including critical genes, pathways and their interactions, require investigation.

In the present study, microarray analysis was performed for the screening of differentially expressed genes (DEGs) between microbiota-depleted mice and control mice. Subsequently, functional and pathway enrichment analyses of the DEGs were processed. Finally, literature associated with the DEGs was mined and their associations were analyzed. The results of the present study may identify potential important genes for further investigations on intestinal flora and human health. 
A

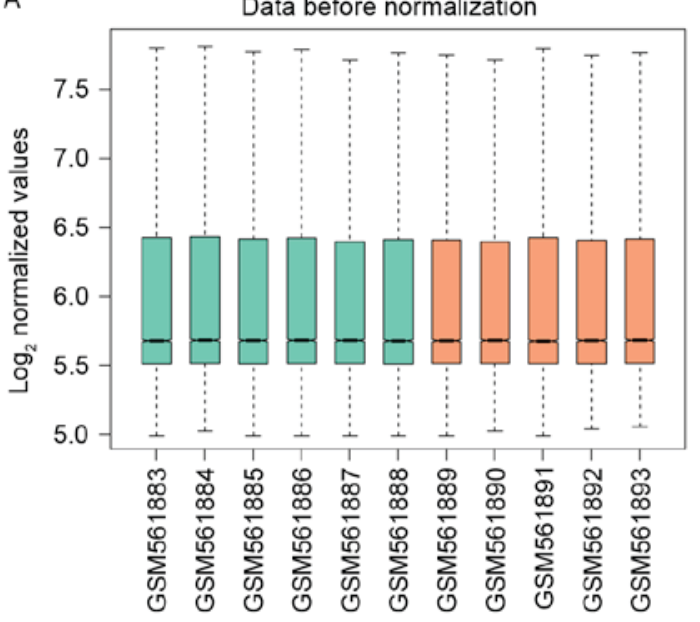

B

B Data after normalization

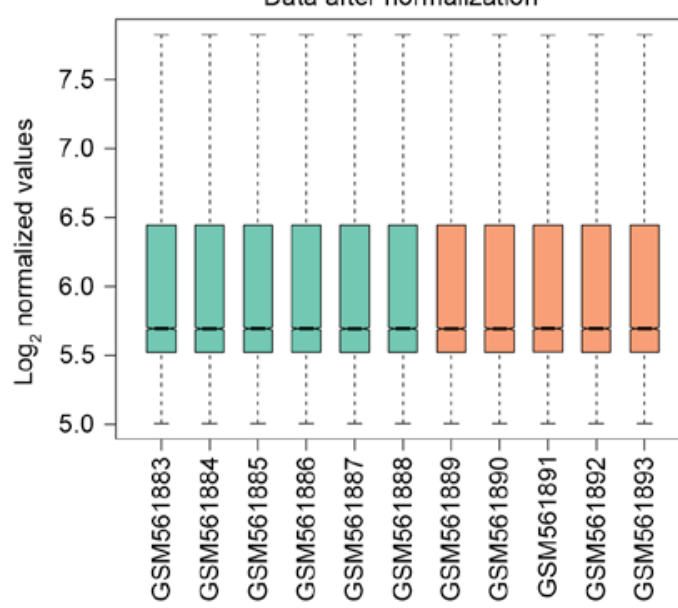

$\square$ Control mice

๑ Microbiōta_depleted_mice

Figure 1. Box-plot of raw data standardization. Data are shown (A) prior to and (B) following normalization. Sample names are on the horizontal axis and the logarithm transformations of the expression values are shown on the vertical axis.

\section{Materials and methods}

Data acquisition. The gene expression profiles of GSE22648 were downloaded from the Gene Expression Omnibus database (www.ncbi.nlm.nih.gov/geo) with the platform GPL6887 (Illumina MouseWG-6 v2.0 expression beadchip) (10). A total of 11 colon intestinal epithelial cell samples were obtained from mice, including five microbiota-depleted mice and six control mice, and analyzed.

Data preprocessing and DEG screening. The Series Matrix File (ftp.ncbi.nlm.nih.gov/geo/series/GSE22nnn/GSE22648/matrix) was downloaded to transfer probe names into gene symbols based on the platform annotation information. Using the aggregate function of the R statistical package, version 3.4.0 (stat.ethz. $\mathrm{ch} / \mathrm{R}$-manual/R-devel/library/stats/html/aggregate.html), the mean value was considered to be the expression value of a gene when multiple probes mapped to a single gene. For probes with missing values, nearest neighbor averaging (11) in the impure package (12) of $\mathrm{R}$ was used for offset of the margin value $(\mathrm{k}$ value was defaulted to 10). Quantile normalization was applied for standardization based on the preprocessCore package of $\mathrm{R}$. The standard matrix was then obtained.

The DEGs between two groups were screened using the Limma package (13) (bioconductor.org/packages/release/ bioc/html/limma.html; version 3.5) and the differences of mean expression values were assessed using the unpaired t-test method. In addition, the Benjamini-Hochberg algorithm (14) was used for p-value correction. Finally, the thresholds of the DEGs were $\log _{2}$ fold change $\log _{2} \mathrm{FCl}>0.585$ and adjusted $\mathrm{p}$-value of $\mathrm{P}<0.05$.

Functional and pathway enrichment of DEGs. The online tool of the Database of Annotation, Visualization and Integrated Discover (DAVID) (15) was used for Gene Ontology (GO; www.geneontology.org) and Kyoto Encyclopedia of Genes and Genomes (KEGG; www.genome.jp/kegg) pathway enrichment analysis of DEGs. The cut-off criterion was $\mathrm{P}<0.05$.
Mining of DEG-associated literature. The DEG-associated literature was mined using the GenCLip 2.0 online tool (ci.smu.edu.cn) (16). The Gene Cluster with Literature Profiles module generated statistically overrepresented key words grouped by a fuzzy cluster algorithm to annotate the input genes. The key words were generated based on the occurrence frequencies of free terms in the gene-associated literature or were provided by the user. The associations among the genes and keywords were linked to the relevant MEDLINE abstracts in which the co-occurrence of genes and keywords are highlighted. The Literature Mining Gene Networks module was used to construct a gene-network of the input genes and generate sub-networks based on the user defined query terms. It also calculated the probability of the random occurrence of the networks through random simulation, and provided GO and pathway enrichment analyses of genes. Co-citation networks of DEGs were constructed for the mining of 'hot genes' which were observed to be repeatedly associated with the other genes. At the same time, the DEGs were clustered to mine the functional information of key genes in previous studies with thresholds of $\mathrm{P}<0.0001$ and hit $\geq 4$.

\section{Results}

Data preprocessing and DEG screening. Following data preprocessing, a total of 30,865 expression data of transcripts were obtained from 11 samples. As shown in Fig. 1, the median of each sample was at the same level.

A total of 115 DEGs were screened with the thresholds of $\mid \log _{2} \mathrm{FCl}>0.585$ and adjusted $\mathrm{p}$-value of $\mathrm{P}<0.05$, which included 58 upregulated genes and 57 downregulated genes. The cluster heatmap of the DEGs is shown in Fig. 2.

GO functional and KEGG pathway enrichment analysis. As shown in Table I, the screened upregulated genes were significantly enriched into various GO terms, including microsome, oxidation reduction and heme binding. They were also enriched in the pathway of drug metabolism. The 
Table I. Top five GO and pathway enrichment terms of differentially expressed genes.

\begin{tabular}{|c|c|c|c|}
\hline Ontology & Term & Count & P-value \\
\hline \multicolumn{4}{|l|}{ Upregulated } \\
\hline $\mathrm{GO}-\mathrm{CC}$ & GO:0005792 microsome & 6 & 5.59E-05 \\
\hline $\mathrm{GO}-\mathrm{CC}$ & GO:0042598 vesicular fraction & 6 & $6.56 \mathrm{E}-05$ \\
\hline $\mathrm{GO}-\mathrm{CC}$ & GO:0005624 membrane fraction & 8 & $1.62 \mathrm{E}-04$ \\
\hline $\mathrm{GO}-\mathrm{CC}$ & GO:0005626 insoluble fraction & 8 & $2.01 \mathrm{E}-04$ \\
\hline $\mathrm{GO}-\mathrm{CC}$ & GO:0000267 cell fraction & 8 & $4.21 \mathrm{E}-04$ \\
\hline GO-BP & GO:0055114 oxidation reduction & 8 & 7.98E-04 \\
\hline GO-MF & GO:0020037 heme binding & 7 & 8.59E-07 \\
\hline GO-MF & GO:0046906 tetrapyrrole binding & 7 & $1.14 \mathrm{E}-06$ \\
\hline GO-MF & GO:0009055 electron carrier activity & 7 & $6.14 \mathrm{E}-06$ \\
\hline GO-MF & GO:0070330 aromatase activity & 4 & $6.55 \mathrm{E}-05$ \\
\hline GO-MF & GO:0005506 iron ion binding & 7 & $1.21 \mathrm{E}-04$ \\
\hline KEGG_PATHWAY & mmu00982:Drug metabolism & 4 & 0.00128 \\
\hline \multicolumn{4}{|l|}{ Downregulated } \\
\hline GO-BP & GO:0006323 DNA packaging & 6 & 7.41E-06 \\
\hline GO-BP & GO:0006334 nucleosome assembly & 5 & 4.43E-05 \\
\hline GO-BP & GO:0031497 chromatin assembly & 5 & 4.93E-05 \\
\hline GO-BP & GO:0034728 nucleosome organization & 5 & 5.19E-05 \\
\hline GO-BP & GO:0065004 protein-DNA complex assembly & 5 & $5.19 \mathrm{E}-05$ \\
\hline $\mathrm{GO}-\mathrm{CC}$ & GO:0000786 nucleosome & 5 & $3.96 \mathrm{E}-05$ \\
\hline $\mathrm{GO}-\mathrm{CC}$ & GO:0044427 chromosomal part & 8 & $4.92 \mathrm{E}-05$ \\
\hline $\mathrm{GO}-\mathrm{CC}$ & GO:0032993 protein-DNA complex & 5 & 8.38E-05 \\
\hline $\mathrm{GO}-\mathrm{CC}$ & GO:0005694 chromosome & 8 & $1.46 \mathrm{E}-04$ \\
\hline $\mathrm{GO}-\mathrm{CC}$ & GO:0000785 chromatin & 6 & $1.51 \mathrm{E}-04$ \\
\hline KEGG_PATHWAY & mmu00590:Arachidonic acid metabolism & 4 & 0.00171 \\
\hline KEGG_PATHWAY & mmu05322:Systemic lupus erythematosus & 4 & 0.00318 \\
\hline KEGG_PATHWAY & mmu00591:Linoleic acid metabolism & 3 & 0.00792 \\
\hline
\end{tabular}

GO, Gene Ontology; CC, cellular component; BP, biological process; MF, molecular function; KEGG, Kyoto Encyclopedia of Genes and Genomes.

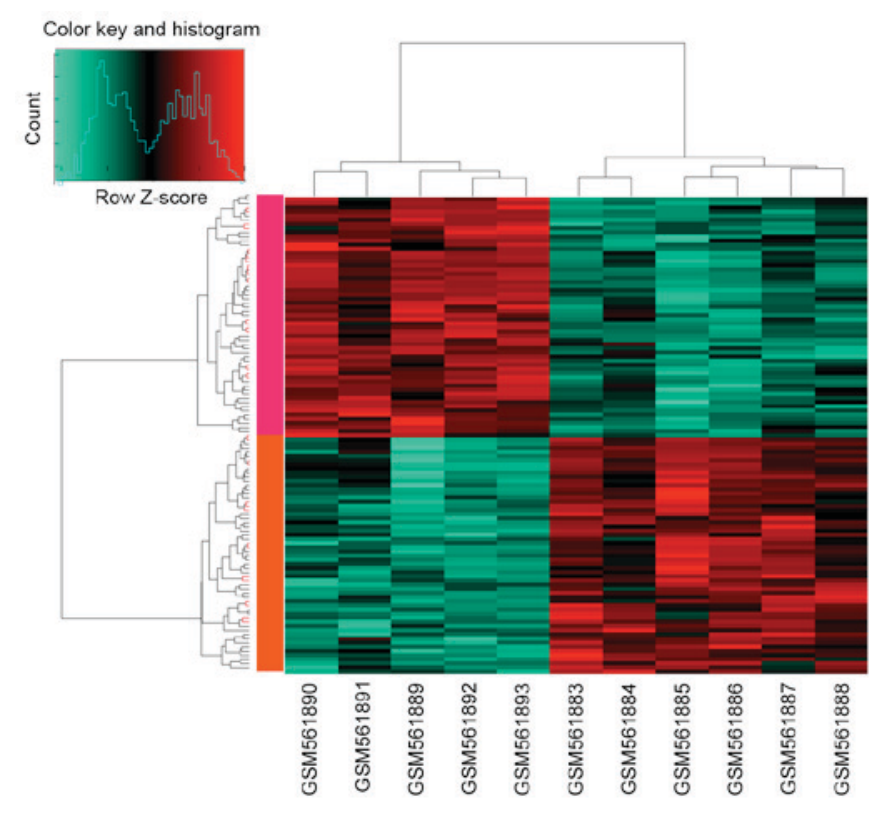

Figure 2. Cluster heatmap of differently expressed genes and samples.
57 downregulated DEGs were enriched in different functions, including DNA packaging, nucleosome assembly and nucleosome. These downregulated DEGs were also enriched in pathways, including arachidonic acid metabolism, systemic lupus erythematosus and linoleic acid metabolism.

DEG-associated literature mining. By mining the citation associations between the DEGs in PubMed, at least eight genes were found to have pairwise co-citation associations. In addition, four upregulated DEGs and four downregulated DEGs were identified with high co-citation levels (Fig. 3 and Table II). Therefore, four downregulated DEGs, including baculoviral IAP repeat containing 5 (BIRC5), aurora kinase A (AURKA), aurora kinase $\mathrm{B}(A U R K B)$ and inner centromere protein antigens $135 / 155 \mathrm{kDa}(I N C E N P)$ were co-cited in several studies. The four upregulated genes were laminin $\alpha 3$, bone morphogenetic protein 1, solute carrier family 22 (organic cation/zwitterion transporter), member 4 (SLC22A4) and PDZ domain containing 3 .

Gene cluster analysis with the literature profiles of all DEGs was processed (Fig. 4 and Table III). The results showed that 

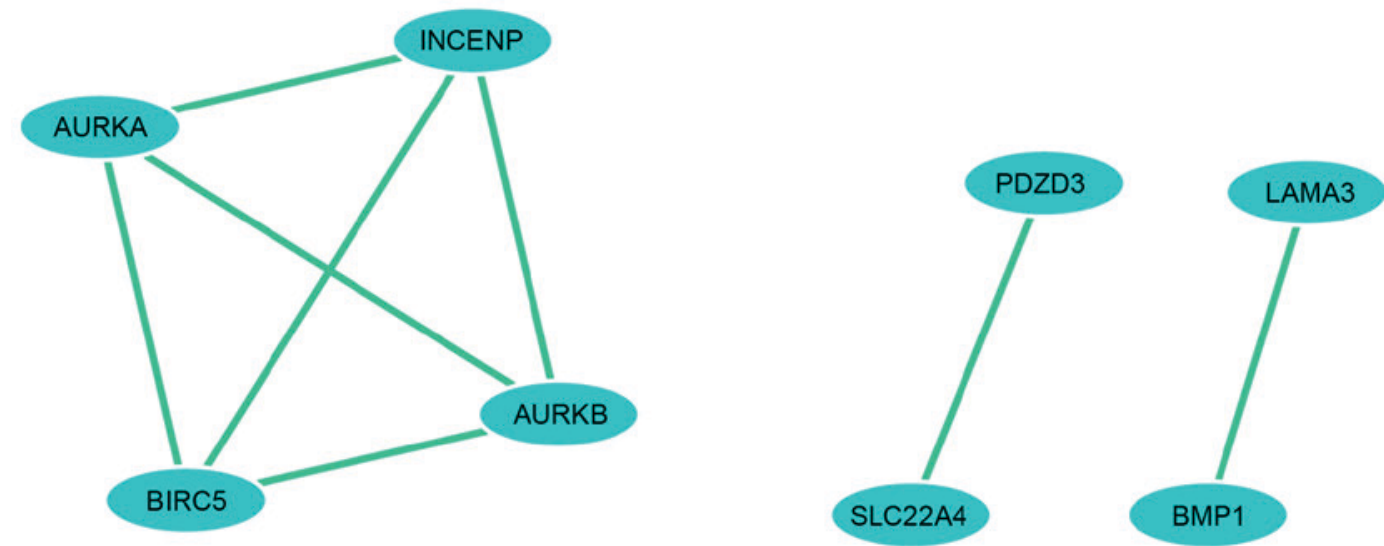

Figure 3. Co-citation network of differently expressed genes. BIRC5, baculoviral IAP repeat containing 5; AURK, aurora kinase; ICENP, inner centromere protein antigens; LAMA3, laminin $\alpha 3$; BMP1, bone morphogenetic protein 1; SLC22A4, solute carrier family 22 (organic cation/zwitterion transporter), member 4; PDZ3, PDZ domain containing 3. The lines represent the co-citation relationship of two genes.

Table II. Results of literature-associated mining of differentially expressed genes.

\begin{tabular}{lcrrr}
\hline Gene & $\begin{array}{c}\text { Co-genes } \\
(\mathrm{n})\end{array}$ & $\begin{array}{c}\text { Co-cites } \\
(\mathrm{n})\end{array}$ & $\begin{array}{c}\text { Total } \\
(\mathrm{n})\end{array}$ & $\begin{array}{c}\log _{2} \text { fold } \\
\text { change }\end{array}$ \\
\hline BIRC5 & 3 & 143 & 5,645 & -0.971 \\
AURKA & 3 & 114 & 990 & -1.054 \\
AURKB & 3 & 169 & 537 & -0.700 \\
INCENP & 3 & 127 & 247 & -0.710 \\
LAMA3 & 1 & 1 & 301 & 0.896 \\
BMP1 & 1 & 1 & 445 & 0.663 \\
SLC22A4 & 1 & 2 & 271 & 0.790 \\
PDZD3 & 1 & 2 & 27 & 0.715 \\
\hline
\end{tabular}

BIRC5, baculoviral IAP repeat containing 5; AURK, aurora kinase; ICENP, inner centromere protein antigens; LAMA3, laminin $\alpha 3$; $B M P 1$, bone morphogenetic protein 1; SLC22A4, solute carrier family 22 (organic cation/zwitterion transporter) member 4; PDZ3, PDZ domain containing 3.

a total of 19 genes, including BIRC5, AURKB, angiotensin I converting enzyme 2 and free fatty acid receptor 2 , were enriched in four modules, including cell division, chromosome segregation, inflammatory bowel disease and inflammatory response. In addition, these modules were associated with various functional terms, including inflammatory response, chromosome condensation, chromosomal passenger complex and phospholipase A2.

\section{Discussion}

Gut bacteria and their reactions are important in gut health and general human health (17). Understanding the process and potential mechanism of the effect of microbial intestinal metabolism on human health is important for the treatment of intestinal diseases (18). In the present study, four upregulated DEGs and four downregulated DEGs were identified with high co-citation levels. Among these genes, the AURKA, INCENP, $B I R C 5$ and AURKB downregulated genes and SLC22A4 upregulated gene were important DEGs, which were enriched in four modules, including cell division, chromosome segregation, inflammatory bowel disease and inflammatory response.

A previous study indicated that intestinal cell proliferation is associated with epithelial homeostasis, regeneration (19), and various diseases, including obstructive jaundice (20) and cancer (4). In the present study, AURKA was identified with a high number of citations on literature mining. This gene encodes a protein, which is a cell cycle regulated kinase, and is also closely associated with chromosome segregation (21). Ratushny et al (22) suggested that by combining with AURKA, SRC kinase was activated and further affected various biological processes, including tumor invasion. In addition, AURKA locates at the spindle poles and regulates cytokinesis by regulating centrosome maturation (23). In the colon of HNF $4 \alpha$ mutant mice, the expression of AURKA was significantly upregulated, and $\mathrm{HNF} 4 \alpha$ was a key regulator for ion transport in the gut mucosa. Therefore, the findings of the present study suggested that AURKA may be involved in the functional modules of chromosome segregation and cell division, and may be important in affecting intestinal microbiome homeostasis in intestinal health.

AURKA is concentrated primarily at the spindle poles in the period of meiosis (MI) and meiosis II, whereas AURKB locates at kinetochores in the period of MI (24). As with $A U R K A, A U R K B$ was identified in the present study as being enriched in functions of chromosome segregation and cell division. Fenton et al (25) processed a microarray analysis, and showed that leptin may upregulate the expression of $A U R K B$ and further induce colon epithelial cell proliferation. In addition, the expression of $A U R K B$ can be regulated by microRNA (miR)-24 by recognizing complementary sequences (26). In addition, in irritable bowel syndrome, miR-24 has been confirmed to increase gut permeability (27). Therefore, $A U R K B$ may also be a target in the treatment of intestinal disease.

INCENP is a complex partner of AURKB for kinase activation, and the regulation of chromosome segregation and cytokinesis (28). In the present study, INCENP was screened to have a high number of citations, and was enriched in cell division and chromosome segregation. INCENP has also been 
Table III. Modules of literature-associated differentially expressed genes.

\begin{tabular}{|c|c|c|c|}
\hline Key word & Hit & Q-value & Genes \\
\hline \multicolumn{4}{|l|}{ \#single1 Enrichment Score: 6.21} \\
\hline INFLAMMATORY RESPONSE & 17 & 0.000223 & $\begin{array}{l}\text { ACE2; BIRC5; CD177; CD276; CHAC1; CYP4B1; } \\
\text { F2R; FFAR2; GPX2; IRF8; MYLK; NFKBIZ; } \\
\text { PLA2G2A;REG3G; RETNLB; SAA1; SAA2 }\end{array}$ \\
\hline \multicolumn{4}{|l|}{ \#cluster1 Enrichment Score: 6.17} \\
\hline CHROMOSOME CONDENSATION & 6 & 0.000023 & $\begin{array}{l}\text { AURKB; BIRC } 5 ; \text { HIFO; HIST2H } 2 B E ; \text { INCENP; } \\
\text { NCAPH }\end{array}$ \\
\hline CHROMOSOME SEGREGATION & 7 & 0.000622 & $\begin{array}{l}\text { AURKA; AURKB; BIRC5; GSG2; HIST2H2BE; } \\
\text { INCENP; NCAPH }\end{array}$ \\
\hline CELL DIVISION & 14 & 0.000568 & $\begin{array}{l}\text { ACE2; AURKA; AURKB; BIRC5; CD177; FBXO6; } \\
\text { FNDC } 3 B ; G S G 2 ; H 1 F O ; H I S T 2 H 2 B E ; \text { INCENP; } \\
\text { MYLK; NCAPH; SIDT1 }\end{array}$ \\
\hline \multicolumn{4}{|l|}{ \#cluster2 Enrichment Score: 5.66} \\
\hline CHROMOSOMAL PASSENGER COMPLEX & 5 & 0.000016 & AURKB; BIRC5; GSG2; INCENP; NBL1 \\
\hline SPINDLE ASSEMBLY CHECKPOINT & 5 & 0.002422 & AURKA; AURKB; BIRC5; GSG2; INCENP \\
\hline SPINDLE CHECKPOINT & 5 & 0.003230 & AURKA; AURKB; BIRC5; GSG2; INCENP \\
\hline \multicolumn{4}{|l|}{ \#single2 Enrichment Score: 5.42} \\
\hline INFLAMMATORY BOWEL DISEASE & 9 & 0.000590 & $\begin{array}{l}\text { AGR2; FFAR2; GPX2; MYLK; NFKBIZ; REG3G; } \\
\text { RETNLB; SAA1; SLC22A4 }\end{array}$ \\
\hline \multicolumn{4}{|l|}{ \#single3 Enrichment Score: 4.73} \\
\hline PHOSPHOLIPASE A2 & 6 & 0.002282 & $\begin{array}{l}\text { CD177; MYLK; PLA2G2A; PLA2G3; SAA1; } \\
\text { SMPDL3B }\end{array}$ \\
\hline
\end{tabular}

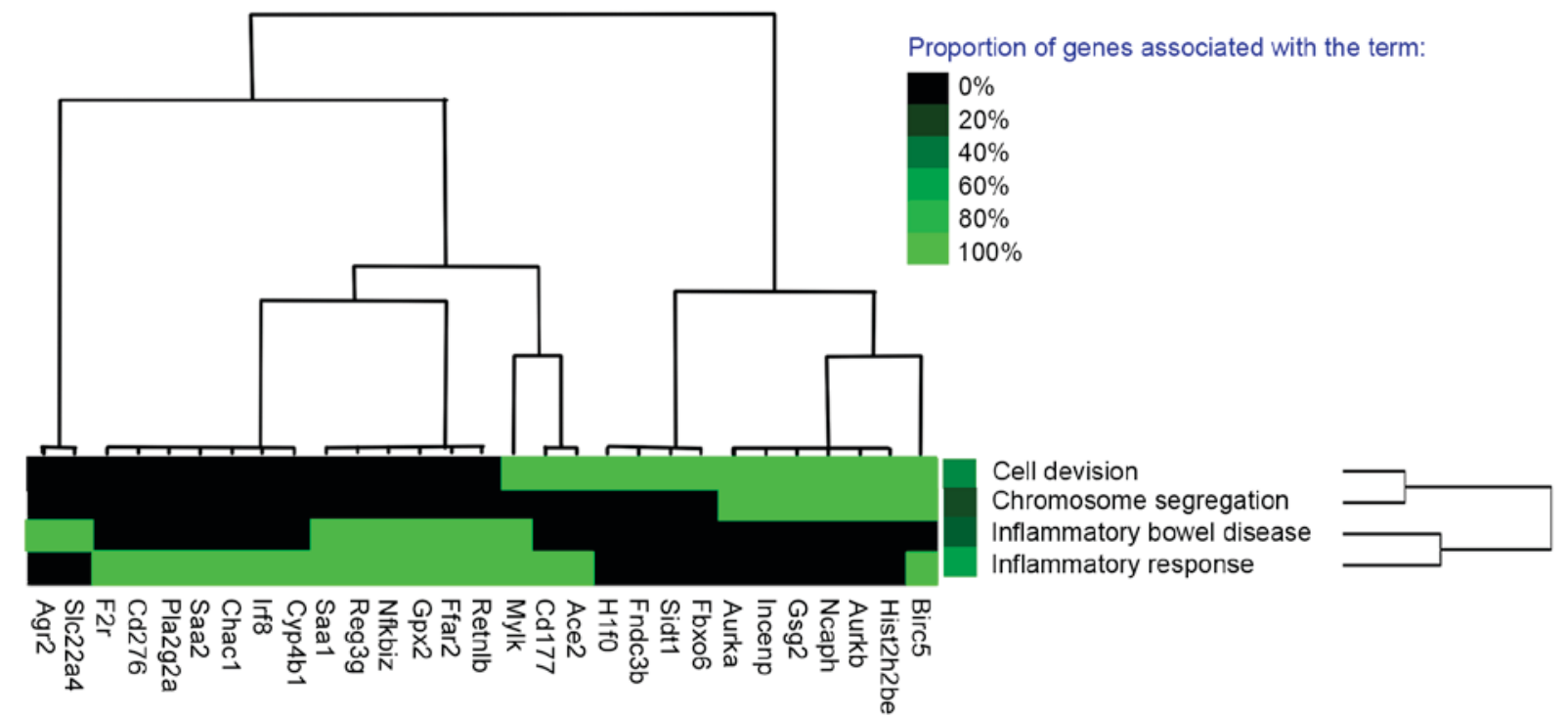

Figure 4. Cluster heatmap of differently expressed genes from literature mining.

confirmed to be an important member of the chromosomal passenger complex, which is an essential regulator for the completion of cell division (29). In addition, INCENP/Sli15 phosphorylation can activate Aurora B/IpI1, which is important for the activity of the chromosomal passenger complex (30). The expression of INCENP has also been shown to be increased in several types of cancer, including colorectal cancer (31). Therefore, the present study hypothesized that INCENP may affect intestinal health through interacting with $A U R K B$.

In the present study, BIRC5 was enriched in three modules, including cell division, chromosome segregation and inflammatory response. Neufert et al (32) confirmed that BIRC5 
can limit bacterial growth and is involved in mucosal wound healing, which suggests a promising therapeutic approach for disturbed intestinal homeostasis. In addition, activated signal transducer and activator of transcription 3 can induce the transcription of $B I R C 5$ and regulate tissue defense during the process of gastrointestinal infection. Previous studies have also shown that microbiota and commensal bacteria can induce inflammatory response in immunocompromised hosts (33), and when the normal microbial community is disrupted, the risk of inflammation is increased (34), This offers novel insights into the pathogenesis of disease for treating intestinal disorders. The gut microbiota can also affect host metabolism and produce various pro-inflammatory molecules, including lipopolysaccharide, assisting in the development of treatments for metabolic diseases (35). Therefore, BIRC5 may be a critical gene in the effects of microbiota on intestinal health.

Of the upregulated DEGs identified in the present study, $S L C 22 A 4$ was found to have a high number of citations and was enriched in the module of inflammatory bowel disease. A mutation of SLC22A4, which encodes organic carnitine transporter 1 , has been confirmed to have a clinical role in gut health (36). In addition, Tokuhiro et al (37) showed that the organic cation transporter SLC22A4 functioned as a transporter in the inflammatory milieu. A previous study also confirmed that polymorphisms of SLC22A4 were closely associated with early inflammatory bowel disease (38). The development of the gut microbiota is essential for promoting the gut immune system and maintaining system balance (39). Therefore, SLC22A4 may be an essential gene for intestinal health via modulating the immune system.

In conclusion, the present study performed bioinformatics analysis of the effects of gut flora on molecular changes in intestinal epithelial cells. AURKA, INCENP, BIRC5, AURKB and $S L C 22 A 4$ were identified as potential target genes for intestinal disease treatment through their involvement in various functions, including cell division, chromosome segregation, inflammatory bowel disease and inflammatory response. These results indicate novel therapeutic targets for diseases caused by disturbed intestinal homeostasis. However, there were limitations to the present study; gut microbiota are associated with the delivery mode and the type of feeding in infants, and changes throughout life between infancy and elderly. Therefore, further experiments are required to confirm the results obtained in the present study.

\section{References}

1. Abreu MT, Fukata M and Arditi M: TLR signaling in the gut in health and disease. J Immunol 174: 4453-4460, 2005.

2. Jeurissen SH, Lewis F, van der Klis JD, Mroz Z, Rebel JM and Ter Huurne AA: Parameters and techniques to determine intestinal health of poultry as constituted by immunity, integrity and functionality. Curr Issues Intest Microbiol 3: 1-14, 2002.

3. Ott SJ, Musfeldt M, Wenderoth DF, Hampe J, Brant O, Fölsch UR, Timmis KN and Schreiber S: Reduction in diversity of the colonic mucosa associated bacterial microflora in patients with active inflammatory bowel disease. Gut 53: 685-693, 2004.

4. Round JL and Mazmanian SK: The gut microbiota shapes intestinal immune responses during health and disease. Nat Rev Immunol 9: 313-323, 2009.

5. Qin J, Li R, Raes J, Arumugam M, Burgdorf KS, Manichanh C, Nielsen T, Pons N, Levenez F, Yamada T, et al: A human gut microbial gene catalogue established by metagenomic sequencing. Nature 464: 59-65, 2010.
6. Guarner F and Malagelada JR: Gut flora in health and disease. Lancet 361: 512-519, 2003.

7. Jia ZY, Xia Y, Tong D, Yao J, Chen HQ and Yang J: Module-based functional pathway enrichment analysis of a protein-protein interaction network to study the effects of intestinal microbiota depletion in mice. Mol Med Rep 9: 2205-2212, 2014.

8. Smith AG, O'Doherty JV, Reilly P, Ryan MT, Bahar B and Sweeney T: The effects of laminarin derived from Laminaria digitata on measurements of gut health: Selected bacterial populations, intestinal fermentation, mucin gene expression and cytokine gene expression in the pig. Br J Nutr 105: 669-677, 2011.

9. Song $M$ and Kellum JA: Interleukin-6. Crit Care Med 33 (12 Suppl): S463-S465, 2005.

10. Reikvam DH, Erofeev A, Sandvik A, Grcic V, Jahnsen FL, Gaustad P, McCoy KD, Macpherson AJ, Meza-Zepeda LA and Johansen FE: Depletion of murine intestinal microbiota: Effects on gut mucosa and epithelial gene expression. PLoS One 6: e17996, 2011.

11. Altman NS: An introduction to kernel and nearest-neighbor nonparametric regression. The American Statistician 46: 175-185, 1992.

12. Hastie T, Tibshirani R, Narasimhan B and Gilbert C: Impute: Imputation for microarray data. Bioinformatics 17: 520-525, 2001.

13. Shabalin AA, Tjelmeland H, Fan C, Perou CM and Nobel AB: Merging two gene-expression studies via cross-platform normalization. Bioinformatics 24: 1154-1160, 2008.

14. Rudy J and Valafar F: Empirical comparison of cross-platform normalization methods for gene expression data. BMC Bioinformatics 12: 467, 2011.

15. Leek JT, Johnson WE, Parker HS, Fertig EJ, Jaffe AE, Zhang Y, Torres LC and Storey JD: sva: Surrogate Variable Analysis. R package version 3, 2013.

16. Wang JH, Zhao LF, Lin P, Su XR, Chen SJ, Huang LQ, Wang HF, Zhang H, Hu ZF, Yao KT and Huang ZX: GenCLiP 2.0: A web server for functional clustering of genes and construction of molecular networks based on free terms. Bioinformatics 30 : 2534-2536, 2014.

17. Puupponen-Pimiä R, Aura AM, Oksman-Caldentey KM, Myllärinen P, Saarela M, Mattila-Sandholm T and Poutanen K: Development of functional ingredients for gut health. Trends Food Sci Technol 13: 3-11, 2002.

18. Pérez-Cobas AE, Gosalbes MJ, Friedrichs A, Knecht H, Artacho A, Eismann K, Otto W, Rojo D, Bargiela R, von Bergen M, et al: Gut microbiota disturbance during antibiotic therapy: A multi-omic approach. Gut 62: 1591-1601, 2013.

19. Barker N: Adult intestinal stem cells: Critical drivers of epithelial homeostasis and regeneration. Nat Rev Mol Cell Biol 15: 19-33, 2014.

20. Assimakopoulos SF, Tsamandas AC, Louvros E, Vagianos CE, Nikolopoulou VN, Thomopoulos KC, Charonis A and Scopa CD: Intestinal epithelial cell proliferation, apoptosis and expression of tight junction proteins in patients with obstructive jaundice. Eur J Clin Invest 41: 117-125, 2011.

21. Ding J, Swain JE and Smith GD: Aurora kinase-A regulates microtubule organizing center (MTOC) localization, chromosome dynamics, and histone-H3 phosphorylation in mouse oocytes. Mol Reprod Dev 78: 80-90, 2011.

22. Ratushny V, Pathak HB, Beeharry N, Tikhmyanova N, Xiao F, Li T, Litwin S, Connolly DC, Yen TJ, Weiner LM, et al: Dual inhibition of SRC and Aurora kinases induces postmitotic attachment defects and cell death. Oncogene 31: 1217-1227, 2012.

23. Nikonova AS, Astsaturov I, Serebriiskii IG, Dunbrack Jr RL and Golemis EA: Aurora A kinase (AURKA) in normal and pathological cell division. Cell Mol Life Sci 70: 661-687, 2013.

24. Shuda K, Schindler K, Ma J, Schultz RM and Donovan PJ: Aurora kinase B modulates chromosome alignment in mouse oocytes. Mol Reprod Dev 76: 1094-1105, 2009.

25. Fenton JI, Lavigne JA, Perkins SN, Liu H, Chandramouli GV, Shih JH, Hord NG and Hursting SD: Microarray analysis reveals that leptin induces autocrine/paracrine cascades to promote survival and proliferation of colon epithelial cells in an Apc genotype-dependent fashion. Mol carcinog 47: 9-21, 2008.

26. Felekkis K, Touvana E, Stefanou $C$ and Deltas C: microRNAs: A newly described class of encoded molecules that play a role in health and disease. Hippokratia 14: 236-240, 2010.

27. Vicario M, Martínez C and Santos J: Role of microRNA in IBS with increased gut permeability. Gut 59: 710-712, 2010.

28. Honda R, Körner R and Nigg EA: Exploring the functional interactions between Aurora B, INCENP, and survivin in mitosis. Mol Biol Cell 14: 3325-3341, 2003. 
29. Guta FF, O'Tooleb P and Walsha MA: MS04 P01 Structural and functional studies of the probiotic organism Lactobacillus salivarius Mario Bumanna, Heinz. Acta Cryst 63: s127, 2007.

30. Yang J, Zappacosta F, Annan RS, Nurse K, Tummino PJ, Copeland RA and LaiZ: The catalytic role of INCENP in Aurora B activation and the kinetic mechanism of Aurora B/INCENP. Biochem. J 417: 355-360, 2009.

31. Chang JL, Chen TH, Wang CF, Chiang YH, Huang YL, Wong FH, Chou CK and Chen CM: Borealin/Dasra B is a cell cycle-regulated chromosomal passenger protein and its nuclear accumulation is linked to poor prognosis for human gastric cancer. Exp Cell Res 312: 962-973, 2006.

32. Neufert C, Pickert G, Zheng Y, Wittkopf N, Warntjen M, Nikolaev A, Ouyang W, Neurath MF and Becker C: Activation of epithelial STAT3 regulates intestinal homeostasis. Cell Cycle 9: 652-655, 2010

33. Hooper LV, Littman DR and Macpherson AJ: Interactions between the microbiota and the immune system. Science 336: 1268-1273, 2012.

34. Kamada N, Seo SU, Chen GY and Núñez G: Role of the gut microbiota in immunity and inflammatory disease. Nat Rev Immunol 13: 321-335, 2013.

35. Tremaroli V and Bäckhed F: Functional interactions between the gut microbiota and host metabolism. Nature 489: 242-249, 2012.
36. Shekhawat PS, Sonne S, Carter AL, Matern D and Ganapathy V: Enzymes involved in L-carnitine biosynthesis are expressed by small intestinal enterocytes in mice: Implications for gut health. J Crohns Colitis 7: e197-e205, 2013.

37. Tokuhiro S, Yamada R, Chang X, Suzuki A, Kochi Y, Sawada T, Suzuki M, Nagasaki M, Ohtsuki M, Ono M, et al: An intronic SNP in a RUNX1 binding site of SLC22A4, encoding an organic cation transporter, is associated with rheumatoid arthritis. Nat Genet 35: 341-348, 2003.

38. Ferraris A, Torres B, Knafelz D, Barabino A, Lionetti P, de Angelis GL, Iacono G, Papadatou B, D'Amato G, Di Ciommo V, et al: Relationship between CARD15, SLC22A4/5 and DLG5 polymorphisms and early-onset inflammatory bowel diseases: An Italian multicentric study. Inflamm Bowel Dis 12: 355-361, 2006.

39. Kelly D, King T and Aminov R: Importance of microbial colonization of the gut in early life to the development of immunity. Mutat Res 622: 58-69, 2007.

This work is licensed under a Creative Commons Attribution-NonCommercial-NoDerivatives 4.0 International (CC BY-NC-ND 4.0) License. 